\title{
Notes sur la Conférence de Lucknow (Inde) sur l'Élevage dans les régions sous climat tropical et subtropical
}

\author{
par L.-M. FEUNTEUN
}

Une conférence sur l'élevage en milieu tropical et subtropical s'est tenue à Lucknow (Inde) du 13 au 22 février 1950 sous l'égide de la F.A.O.

Étaient représentés à cette conférence les pays suivants : Australie, Belgique, Birmanie, Ceylan, Égypte, France, Inde, Indonésie, Iran, Italie, Pakistan, Royaume Uni, Siam.

$\mathrm{Ne}$ se trouvaient représentés : aucun des états d'Amérique situés sous climat tropical et subtropical, aucun des États d'Afrique du Sud, aucun des territoires espagnols et portugais d'Afrique, aucun des pays du Proche-Orient sous climat subtropical sauf l'Iran; manquaient également, la Chine et la Malaisie. Ceci montre que les absents étaient à la fois nombreux et importants.

Après désignation du Président M. Sardat Dafar Singh, Chef de la délégation indienne, M. R.-W. Phillips, délégué du Directeur de la Division d'Agriculture de la F.A.O., procéda au rappel de quelques notions élémentaires de zootechnie puis formula des propositions pour la conduite des travaux de la conférence:

Io Présentation du matériel de base par les. délégués de la F.A.O.

$2^{\circ}$ Exposé de l'essentiel des rapports établis par les différents territoires représentes à la Conférence.

$3^{\circ}$ Établissement de rapports résumés groupant les points importants notés au cours de la Conférence.

$4^{\circ}$ Préparation de recommandations sur ce qu'il y aurait lieu de réaliser pour faire progresser l'élevage des pays tropicaux.

Pour l'etablissement des rapports et recommandations, trois comités de travail furent constitués :

Comité A : Recherche de génétique et expériences appliquées d'élevage.

Comité B : Recherche de physiologie et son application dans la pratique, y compris les essais sur le terrain. Procédés et méthodes d'évaluation du climat.

Comité $\mathrm{C}$ : Programmes proposés pour généraliser l'application des connaissances nouvelles dans l'amélicration des animaux domestiques.

\section{I. - MATÉRIEL DE BASE}

Il comportait deux rapports destinés à faire, en gros, le point sur la situation actuelle des connaissances en matière de genétique et de physiologie animale.

Nous donnerons un résume de ces deux rapports qui, on le verra, contiennent un certain nomiore de notions déjà classiques ou en passe de le devenir.

Applications de la génétique à l'élevage en milien tropical on subtropical, par R.-W. Phillips.

Les travaux effectués dans différents pays ont conduit aux notions suivantes:

Appréciation des qualités. - Ce facteur est essentiel pour prétendre a l'amélioration des qualités reproductrices des animaux. Les éleveurs ont généralement recours pour cela à l'appréciation visuelle ou tactile: si certains caractères peuvent s'apprécier gràce à un simple examen extérieur, par contre certaines aptitudes comme la production du lait, de viande et les facultés reproductrices ne peuvent être évaluées de cette manière.

Des methodes plus scientifiques ont été adoptées telle que la mesure des caractéristiques des brins de laine, ia mestre des coefficients d'engraissement pour les animaux de boucherie, la mesure de la puissance de traction des chevaux, etc.

Méthodes de sélection. - Trois méthodes de selection sont possibles :

$1^{\circ}$ la méthode du tandem, où la sélection porte successivement sur les différents caractères à améliorer, jusqu'à obtention du résultat global;

$2^{\circ}$ la méthode de la sélection totale où la sélection des divers caractères est recherchée simultanément; 
$3^{\circ}$ la méthode de sélection à niveaux indépendants qui fixe un certain niveau minimum pour chaque caractère, et élimine tout animal restant au-dessous de ce niveau.

Hazel et Lush (1942) ont procédé à l'étude comparative de ces trois méthodes et ont constaté que celle de la sélection totale est la plus efficace et la méthode du tandem la moins bonne.

Appréciation par l'hérédité des caractères. Un géniteur peut être jugé par les caractères de ses ascendants, ou mieux encore par ceux de sa descendance; ce facteur n'est praticable que si le rythme de la reproduction est rapide et si l'apparition des caractères est suffisamment précoce.

Méthodes d'élevage. - Trois méthodes d'amélioration sont possibles:

10 Sélection à l'intérieur d'une race donnée;

$2^{\circ}$ Croisement continu par utilisation d'une race plus productive;

$3^{n}$ Recherche d'un type nouveau en fixant des caractères pris à une race améliorée ou aux deux races croisées.

La sélection à l'intérieur d'une race donnée permet d'exploiter l'adaptation de cette race aux conditions locales et dispense des frais d'importation de gèniteurs étrangers et des inconvénients résultant de l'importation de maladies. Elle a l'inconvénient de ne donner de résultats qu'à longue échéance.

Le croisement continu offre l'avantage de pouvoir améliorer un grand nombre de femelles à partir d'un petit nombre de mâles, et d'aller plus vite.

Dans certains cas, il arrive que des animaux de pur sang importés s'adaptent parfaitement à leur nouveau milieu.

Dans ces conditions, l'amélioration du cheptel d'une grande région pourra être basée sur l'action d'un petit nombre de géniteurs entretenus dans des établissements administratifs d'élevage. La capacité de production d'un troupeau commercial dépendra de I'amélioration continuelle des géniteurs.

Amélioration des animaux domestiques sur le plan national. - Les études sur l'amélioration du bétail n'ont gćnćralement qu'un caractère restreint limité à une fraction de la population. Fait exception à ceci une étude réalisée par le New-Zeland Dairy Board sur l'anlélioration de la production en matière grasse par tête d'animal et par an. Ces études ont montré, abstraction faite des facteurs saisonniers et climatiques, que sur une periode de vingt-quatre ans (1919 à 1943) le relèvement total de la production de matières grasses a été de 61 livres par vache. Toutefois, le rythme de l'accroissement annuel ne fut pas uniforme : de 4 livres par vache au début de J'expérience, ce taux s'abaissa progressivement à trois quarts de livre à la période terminale.

La sélection des filles issues de mères bonnes productrices a donné un résultat décevant, pour trois raisons :

$1^{a}$ les filles issues du croisement d'une mère hautement sélectionnée avec un taureau non amélioré, présentent des qualités à peine supérieures (15\% seulement) aux filles nées de ce meme étalon et d'une mère mauvaise productrice;

$2^{\circ}$ pour 100 vaches mères, on obtenait seulement 32 genisses pour la reproduction et les trois quarts de celles-ci doivent assurer les remplacements dans le troupeau, laissant ainsi un nombre limité de sujets supérieurs aux mères pour le travail de sélection:

$3^{\circ}$ des variations dans la composition numérique et raciale du troupeau initial pendant la période expérimentale.

L'association d'une alimentation améliorée à base de grains et de produits ensilés, jointe à une augmentation de la durée de lactation, semblent être les facteurs les plus importants d'une accroissement de la production.

\section{La physiologie et l'amélioration de l'élevage} tropical, par D.-H.-K. Lee, Professeur de climatologie physiologique, Conseiller de, la F.A.O.

L'auteur signale l'insuffisance de nos connaissances sur la physiologie des animaux en milieu tropical.

Il note les facteurs agissant sur l'animal et les modes de réaction normaux ou pathologiques de l'animal sans réellement sortir des données classiques et insiste sur la nécessité de pousser les études de physiologie pour une conduite plus rationnelle et plus efficace du perfectionnement de l'élevage.

\section{II. - POINTS SAILLANTS DES RAPPORTS ET DES DISCUSSIONS}

Les rapports présentés traitent des conditions dans lesquelles l'élevage, la production des animaux domestiques sont pratiqués dans les différents pays.

Les faits saillants difficiles à classer compte tenu de leur variété se résument comme suit sous forme de notes successives :

\section{A) Application des aptitudes - Mesure des qua- lités.}

Les qualités physiologiques des animaux : résistance à la chaleur; production de lait, de matière grasse, de viande, puissance de traction, utilisation de la ration alimentaire, sont importantes à connaître mais sont généralement mal connues en milieu tropical. Des méthodes pratiques de mesure des diverses qualités font généralement défaut.

Les divers rapports des délégations font ressortir que la sélection et le choix des animaux d'élevage 
sont basés sur la seule appréciation visuelle, sauf dans les cas où cst possible la mesure quantitative de la production en lait, en viande ou en laine.

Les rapports de presque tous les délégués font ressortir l'absence d'épreuves convenables pour mesurer les qualités de traction des animaux.

Quelques essais pratiques aux Indes donnent une idée de la puissance de traction maximum d'un bœuf, sans fournir aucune indication sur la résistance de l'animal et son aptitude à se maintenir en état.

Les mensurations comparees entre bons et mauvais tracteurs font ressortir des différences insignifiantes. Peu de différence morphologique également entre animaux laitiers et animaux de travail. L'étude comparative de la température du corps et de la cadence respiratoire avec un travail constant fait ressortir des différences négligeables entre le début et la fin du travail.

Le délégué de l'Indonésie remarque que si l'on fixe à 100 la capacite de travail du bétail local, les métis (croisement avec du bétail de l'Inde) représentent l'indice 128 et les ongoles 180. Toutefois, aucun détail ne fut donné sur les méthodes de mensuration de la traction.

Le délégué du Pakistan révèle une méthode de mesure du travail qui releve de l'attraction sportive : deux bœufs sont attelés à une lourde charge et sont conduits jusqu'à ce que l'un deux s'écroule de fatigue, ce qui se produit généralement au bout de cinq à dix minutes.

Les différences de conditions entre milieux tropicaux et milieux tempérés montrent que sous les premiers la lactation diminue depuis la promière jusqu'à la troisieme lactation. tandis que l'ordre inverse s'observe dans les pays tempérés.

\section{B) Sélection - Conservation des races locales.}

L'examen des méthodes de reproduction montre que dans de nombreux pays tropicaux se pratique la sélection à rebours : aux Indes et en Égypte, on sacrifie la plupart des bufflons mâles en bas âge. perdant ainsi toute chance de pratiquer une sélection ultérieure. En Indochine, les plus beaux taureaux sont souvent castrés pour faire des bcufs de trait. tandis que les taureaux de petite taille sont gardes pour la reproduction. En Chine, les verrats reproducteurs sont les plus manvais sujets des portées.

L'importation de races étrangères risque dans certains pays d'éliminer la race pure locale. C'est ainsi qu'en Birmanie l'administration a dû crécr un centre d'élevage réserve à la production du bétail local pur, menacé de disparition par importation de sang étranger. Une situation semblable existe a Ceylan, où la race Sinhala locale est entretenue dans deux fermes gouvernementales.

La conférence a souligné l'importance qu'il y a à conserver certaines races locales pures, bien adaptées aux conditions du milieu, telles que le betail Boran dans l'est africain et le bétail Nandi au Kenya. Ces deux races tirent avantage de leur taille réduite et sont relativement bonnes productrices de lait et de viande, de même pour le petit bétail à courtes cornes de Nigeria, la race des lagunes et la race N'Dama des territoires du sud de l'A.O.F. résistants aux trypanosomiases.

Le délégué égyptien signale que les vaches de Damiette produisent 1.500 livres de lait par an, $e$ mème 3.000 livres dans certains troupeaux sélectionnés.

\section{C) Acclimatement - Croisements.}

a) Bovins et bubalins. - En Australie, l'importation, en 1843, de quelques couples de buffles de Timor laissés à l'état sauvage a abouti à une' véritable pullulation de l'espece. On en tue chaque année plus de 20.000 pour exploiter leur peau. Dans ce pays, aucun bétail autochtone n'existait, toute la production animale tire son origine de races importees de Grande-Bretagne. La production commerciale du lait y est poursuivie avec plein succès, au nord du Tropique du Capricorne, sous réserve d'une haute altitude (1.000 mètres). La production de viande, favorisée quantitativement dans le nord de l'île, se heurte aux difficultés de transport vers les abattoirs industriels de la côte. De ce fait, le bétail anglais à membres courts ne convient pas dans cette région en raison de ses possibilités de déplacement réduites.

$L$ aptitude à l'acclimatement, chez la race indienne Red Sindhi, permet à ce bétail de réussir non seulement dans les régions sèches analogues à son habitat naturel, mais encore dans les régions à très hautes précipitations pluviales.

Le Sahiwal, au contraire, se révèle moins favorable aux régions humides. Quant aux essais tentés sur le Tharparkar, ils sont trop récents pour pouvoir faire l'objet de conclusions valables.

Aux Indes, un vaste programme est'à l'étude pour l'obtention d'une race bovine à deux fins (lait et travail); un troupeau initial de 3.500 têtes, composé de Nimari, de Malvi et de Gaolao, sera d'abord amélioré par sélection, puis croisé avec les races Red Sindhi, Sahiwal et Tharparkar. Les caractères obtenus sur les produits seront ultérieurement fixés par consanguinité.

Le zébu malgache importé de l'Inde resscmblo beaucoup à la race Ponwar dont il semble être descendant.

La race bubaline Murrah produit moins de lait à Ceylan que dans l'Inde ou dans le Pakistan.

Les races européennes importées ne s'adaptent à Ceylan que dans les régions situées au-dessus d'une 
altitude de 2.000 mètres et qui représentent seulement $1 \%$ de la surface de l'île. Pour cette raison, le pays se consacre plutôt à l'amélioration du bétail local (Sinhala).

Le délégué de l'Indonésie signale que la race Holstein réussit parfaitement au-dessus de 250 mètres; toutefois, son adaptation est possible au niveau de la mer sous certaines conditions d'entretien (étables fraîches, alimentation appropriee).

Au Kenya, les races européennes réussissent en dilitude (2.000 miètres) à condition qu'elles y trouvent une pluviométrie suffisante. Avec la race Aberdeen Angus, on a pu obtenir sur des bœufs de quatre ans des rendements nets de 300 kilos.

Aux Indes, le croisement des races Frise-Holstein et Jersey avec le bétail local semble donner le maximum de résultats au stade du cinq huitième de sang améliorateur. Les autorités du pays s'efforcent de fixer les caractères de ce croisement de manière à permettre le ravitaillement en lait de certains centres urbains.

Au Pakistan, la race Holstein ne semble pas s'être adaptée correctement aux conditions climatiques du pays. Elle résiste bien aux mois d'hiver, à 2.000 mètres d'altitude, mais la saison d'été est trop chaude pour elle.

A Madagascar, les croisements de la race Zébu locale avec les taureaux normands et Friesland ont donné les meilleurs résultats.

En Afrique Occidentale Française; l'importation de races françaises telles que le Normand et le Charolais n'ont pas donné de résultats satisfaisants en dehors des stations administratives d'élevage.

A Ceylan, le croisement du bétail Sindhi avec certaines races européennes donne de bons résultats à la première génération (vaches du Cap Matton), et de moins bons résultats aux générations suivantes au fur et à mesure que l'on s'éloigne du type indigène.

En Thailland, l'introduction de bétail Holstein, Jersey et Shorthorn a été contrariée par leur manque de faculté d'adaptation. Quelques reproducteurs des Indes ont pu intervenir pour améliorer le format des animaux indigènes.

En Australie, dix-neuf zébus furent importés des U.S.A. en 1933 et placés dans des stations de la zone tropicale. Le résultat obtenu montre que le métis bœuf zébu est plus précoce et fournit une meilleure carćasse que le bétail européen; on attribue ce fait à une plus grande résistance aux tiques.

L'Indochine a récemment procédé à l'importation de cent trente Sindhi, Sahiwal et Tharparkar et de buffles Murrah, en vue de déterminer à la fois leur aptitude à s'adapter aux conditions locales et leur aptitude à amélioror lo bétail local par croisements. b) Ovins. - En Égypte, l'amélioration du mouton de race locale, à grosse queue, au moyen de la race anglaise Suffolk, apporte un accroissement à la fois dans la quantité et dans la qualité de viande et de laine produites.

En Afrique Occidentale Française, les résultats obtenus par l'importation du mérinos ont été décevants; par contrc, lc croisement du mouton maure avec le karakul s'avère satisfaisant, de même qu'en Afrique Équatoriale Française.

L'intruduction du karakul sur le mouton local égyptien donine également des résultats encourageants, que l'on entend poursuivre par croisement continu.

En Iran, la race ovine locale de Lori, croisée avec les mérinos, donne dès la première génération, une production lainière doublée.

Aux Indes, le mouton Hissardale s'adapte bien au Punjab, mais mal dans la région de Kangra où il est soumis à un nomadisme intense. Au Cachemire, les produits issus des brebis locales et des mérinos peuvent, dès la troisième génération, soutenir la comparaison avec le mérinos pur.

Au Pakistan, le mouton karakul est employé avec succès pour améliorer par croisemenl le cheptel ovin des provinces frontières. Le mérinos y a supporté le climat froid et assez humide du district de Hazara. Des essais d'importation de la race Pushti-koh furent interrompus en raison de la difficulté de s'approvisionner en nouveaux géniteurs.

c) Caprins. - Au Pakistan, un gros effort a été entrepris pour améliorer la race indigène des collines avec la chère angora, en vue de développer la production du mohair.

Les résultats, obtenus portent sur la quantité de poils produite (4 livres 25 par an chez les sept huitièmes de sang, contre 1 livre 20 chez: les chèvres locales) et sur la qualité du poil qui, chez les métis sept huitièmes a acquis les caractères de l'angora. En contrepartie, l'infusion de sang angora a déterminé une réduction de là taille de la race Iocale.

A Madagascar, le croisement angora avec la chèvre locale a donné des résultats analogues très satisfaisants.

En Égypte, la chèvre angora croisée avec la chèvre égyptienne détermine, dès la deuxième génération, un pelage fin, ondulé et lustré, où la couleur blanche apparait comme caractère dominant.

A Ceylan, les races caprines anglo-nubiennes, Alandri et Kaomori, ont donné des résultats peu satisfaisants. Par la suite, des chevres de Jamnapari furent importées, mais la plupart succombèrent à une paralysie enzootique de nature inconnue.

$L$ 'influence du climat tropical sur le développement du nanisme animal furent évoqués; le bétail nain 
se rencontre dans les régions basses et humides. Dans cet ordre d'ideee, le délégué de Ceylan rapporte que la race Red Sindhi de la péninisule, introduite à Ceylan, enregistre une diminution de taille dès la deuxieme génération.

\section{D) Nécessité d'étades physiologiques.}

L'obtention de rendements élevés en productions diverses (viande, lait, travail, etc.) sous les tropiques, est subordonnée tout d'abord au choix de races hautement productives, mais aussi à leur aptitude a conserver intact ce taux de productivité.

Sous ce rapport, de nombreux facteurs interviennent pour modifier l'équilibre physiologique de l'animal, tels que : la température élevée, l'état de saturation hygrométrique, la fréquence des affections contagieuses et parasitaires, le régime alimentaire irrégulier, etc.

Ces considérations font ressortir l'intérêt majeur de recherches physiologiques tendant à définir les moyens et le degré d'action de ces divers facteurs.

Il ressort de la discussion qu'une amélioration substantielle des races animales pourra être obtenue lorsque les données physiologiques énoncées ci-dessus auront été précisées.

10 Études expérimentales, - a) En matière de régulation thermique :

L'abaissement de la température du corps peut provenir d'une moindre dépense énergétique, d'une perturbation du métabolisme, d'un dérèglement de certaines glandes endocrines, de la mise au repos prolongé, etc.

Certains animaux comme les chiens realisent leur équilibre thermique par échanges avec l'extérieur au niveau de leur arbre respiratoire. Mais ces sujets risquent de perdre, de ce fait, trop d'acide carbonique, et de troubler ainsi leur équilibre acido-basique.

Dans la plupart des races, l'équilibre thermique est assuré au niveau de la peau elle-même, par divers procédés tels que : circulation sanguine capillaire, pouvoir tsolant du tégument, capacité d'absorption due à la présence de pigment, pouvoir de rayonnement, etc.

Les méthodes de mesure de l'irrigation sanguine sont rudimentaires. Celles qui mesurent le pouvoir isolant de la robe ont besoin d'être précisées.

Le phénomène de régulation thermique peut mettre à rude épreuve certains systemes endocriniens tels que : pituitaire, thyroïde, glandes génitales, surrénales, etc. Des études sur le comportemtcrt de ces systemes glandulaires en rapport avec l'action de la chaleur conduiraient, sans aucun doute, à des techniques fort compliquées, mais d'urr intérêt primordial. b) En matiere de nutrition:

Ia plupart des études sur la nutrition des animaux ont ete jusqu'ici conduites en milieux tempérés. Sous les tropiques, des facteurs differents interviennent : modification du régime alimentaire, changement des besoins nutritifs de l'animal, fonctionnement différent du métabolisme alimentaire, etc.

D'où la nécessité de reconsidérer la physiologie de la nutrition en se plaçant dans les conditions tropicales.

$2^{\circ}$ Études sur le terrain. - Ces etudes, marchant de pair avec les spéculations de laboratoire, peuvent apporter une importante contribution à la connaissance du comportement physiologique des diverses races.

Recherches en cours. - Des recherches sont en cours dans ces deux domaines :

a) Sur le plan expérimental.

Des chambres de psychometrie ont été installées pour la mesure des réactions du bétail zébu aux differentes conditions atmosphériques (température et humidité) pour l'étude de l'amplitude du métábolisme.

Ces recherches sont conduites, en Arriérique par Mitchell et Hamilton à l'Universite agricole de l'mlinois, par Kleiber et ses collaborateurs à la station agronomique de Californie. En Australie, par Lee à l'Universite de Queensland.

Findlay a également installé à 'I'Institut d'Ayr (Écosse) une salle de psychométrie pour l'étude du sétail local. Cet Institut doit publier une revue traitant des reactions du bétail aux conditions ciimatiques des pays chauds, basées sur la distribution des glandes sudoripares et des follicules pilelix.

Un projet d'études expérimentales similaires, visant le bétail laitier des Indes; est envisagé par le Bureau de I'Industrie laitière de Beltsville (U.S.A.).

b) Sur le terrain.

Parallelement aux recherches spéculatives cidessus, des études pratiques sur le terrain sont conduites dans certains pays.

Bischop, en Afrique du Sud, a entrepris de déterminer le comportement comparatif du betail local et du bétail métis aux conditions du pays. Les veaux ont déjà pu être classés en tolérants ou non tolérants a la chaleur, en fonction du degré de feutrage de leur pelage.

En Indochine, des recherches ont été faites en vue d'établir la corsélation entre le tégirie des chutes de pluies et l'éclosion des maladies du bétail. Les épizooties apparaigsent le plus souvent au début de la saison des pluies, à l'époque des labours. Le protocole expérimental le plus complet émane de l'Institut Indien de Recherches vétértinaires 
d'Izatnagar qui, depuis. 1936, se consacre à élucider les problèmes suivants :

- variation de la composition du sang sur le bétail laitier, en fonction de variations saisonnières;

- phénomène de polypnée et d'essouflement comme séquelles de la fière aphteuse;

- effet des chutes de pluie froide sur la tolérance du buffle, du zébu, du mouton et du chien à la chaleur;

- effet des bains de boue sur la production laitière des bufflesses;

- incidences des variations saisonnières sur la qualité du sperme de bélier;

- influence de la médication thyroïdienne sur la qualité du sperme;

- effet des conditions de milieu sur l'évolution des maladies non infectieuses;

- étude des causes de dégénérescence des races animales en régions chaudes et humides.

En Australie, enfin, il convient de signaler les recherches sur la corrélation entre le maintien à l'obscurité et la courbe des facultés reproductrices des géniteurs mâles.

Énumération des principaux problèmes à résoudre. - La conférence dresse une liste des principaux problèmes à élucider. Les plus importants sont :

- rapports entre besoins nutritifs et utilisation économique;

- pouvoir réflecteur des robes animales à l'égard des différentes radiations du spectre solaire;

- résistance et conductibilité des diverses robes à la chaleur;

- rôle de l'irrigation sanguine dans la thermorégulation;

- distribution et fonctionnement du système de glandes sudoripares;

- rapports entre métabolisme de l'eau et métabolisme des minéraux;

- fonctionnement de l'émonctoire rénal et troubles de l'élimination urinaire;

- effets photochimiques de la lumière : coup de soleil, accidents de photosensibilisation;

- influence de la lumière sur les fonctions de reproduction.

\section{E) Amélioration des animaux domestiques sous} les tropiques.

Elle est conditionnée par les facteurs suivants : Climat - Alimentation - Pathologie - Facteur humain - Facteur économique - Facteur géographique.

$1^{\circ}$ Climat. - L'introduction de races européennes sous les tropiques est une entreprise difficile et hasardeuse. Dans quelques pays, le choix des hautos altitudes permet de pallier les inconvénients de cette transplantation.

Apres une periode d'adaplation en haules altitudes, les animaux peuvent être transférés progressivement dans les régions moins élevées.

C'est grâce à cet artifice que l'on a pu acclimater des races bovines laitières d'Europe dans des pays tcls que Ceylan, le Konya, Madagascar, Chyprc, ctc.

Par ailleurs, certains pays dotés de climats divers en raison de leur étendue ou de leur disposition géographique pourraient, grâce à cet avantage naturel, maintenir en permanence leurs animaux dans des conditions favorables, grâce à des mouvements, des déplacements réglés sur le rythme des saisons.

$2^{\circ}$ Alimentation. - Dans de nombreux pays, l'élevage d'animaux améliorés est compromis par une sous-alimentation imputable très souvent à l'ignorance des éleveurs, mais aussi, pour certaines régions, à une surcharge inconsidérée des pâtures et à l'absence de réserves fourragères.

La constitution préalable de ressources alimentaires suffisantes apparaît ainsi comme une condition indispensable à toute amélioration du bétail.

Au cours de ces dernières années, la menace de disette qui planait sur certaines populations du globe a conduit les gouvernements à favoriser par des primes spéciales une production accrue des céréales. Les surfaces réservées au bétail se sont, par contrecoup, trouvées réduites dans des proportions équivalentes.

Toutefois, de nombreux pays n'ont pas encore pleinement mis à profit des disponibilités végétales dont ils pourraient disposer en faveur de leur cheptel.

L'Australie se propose dans ce but de développer au maximum la culture du sorgho.

Enfin, l'insuffisance d'eau pour l'abreuvement constitue dans certaines régions un gros obstacle à toute entreprise zootechnique.

$3^{\circ}$ Pathologie. - Ce facteur revêt, dans les régions tropicales et subtropicales, une importance particulièrement décisive.

Les grandes épizooties comme la peste bovine, la péripneumonie, la fièvre aphteuse sévissent encore dans de nombreux pays.

D'autre part, certaines maladies parasitaires comme l'East Coast fever, l'Anaplasmose, la Babesiellose, le Heart Water imposent aux pays intéressés de coûteuses dépenses nécessitées par la destruction des tiques, agents vecteurs de ces maladies.

Dans les régions équatoriales et subéquatoriales d'Afrique, les trypanosomiases. transmises par la mouche tsé-tsé, déciment les troupeaux.

Enfin, sans être aussi spectaculaires que les affections précédentes, toutes les causes de stérilité $\mathrm{du}$ bétail occasionnent de graves dommages à certains élevages de régions tropicales. C'est ainsi que la vaginile et l'épididyrnite contagieuse sévissent 
au Kenya depuis longtemps. Il convient de noter que ces affections n'ont frappé jusqu'ici que les races europeennes, ou leurs croisements, tandis que le bétail strictement local semble dans une certaine mesure réfractaire.

$4^{\circ}$ Facteur humain. - L'homme, par ses méthodes défectueuses, porte une lourde responsabilité dans la mauvaise exploitation de ses ressources animales. En Nigeria, $90 \%$ du bétail sont entre les mains de tribus nomades, qui ne consacrent à leurs animaux aucune culture fourragère.

Dans les Indes, la population manque de substance protéique, cependant que d'immenses ressources en bovins restent inexploitées, conformément aux dogmes de la religion locale qui interdisent l'abatage de cette espèce animale.

En Afrique Noire sévit un grave déséquilibre dans la répartition des ressources alimentaires à base de protéine animale : les régions côtières, fortes consommatrices, sont dépourvues de cheptel, tandis que, plus au nord, des zones surpeuplées d'animaux offrent des surplus considérables et inutilisés.

Enfin, dans presque tous les pays tropicaux, le rythme de l'accroissement des populations indigenes atteint des proportions alarmantes par rapport aux disponibilités alimentaires.

$5^{0}$ Facteurs économique, politique et social. L'élevage de nombreux pays souffre d'investissements financiers insuffisants ainsi que d'une pénurie de personnel qualifié.

Les circonstances économiques propres à chaque pays retentissent sur l'avenir de leur élevage. C'est ainsi qu'en Égypte les travaux d'irrigation en cours doivent fertiliser de grandes étendues de terres, qui scront ensuitc exploitćes par motoculture, entraînant une diminution correspondante de l'élevage.

Dans les Indes au contraire, où la mécanisalion rıe semble point appelée à un avenir immédiat, on continuera à exploiter l'élevage du bœuf, en l'orientant vers une vocation laitière puisque la religion locale interdit son envoi à la boucherie.

La conférence rappelle enfin que l'institution du contrôle des prix et des denrées alimentaires, née des événements politiques de ces dernières années, a constitué dans de nombreux pays un lourd handicap pour l'élevage, par suite des indices rémunérateurs accordés aux céréales de consommation humaine.

$6^{\circ}$ Facteur géographique. - En Australie, dans le Moyen-Orient et dans certaines parties de l'Afrique Noire, de vastes régions propices à l'élevage du bœuf restent inexploitées en raison de leur éloignement de tout moyen de communication.

Sur de vastes régions d'Afrique, la présence de mouches tsé-tsés interdit tout élevage de gros bétail et inflige aux convois d'animaux qui les traversen des pertes considérables.

\section{F) Méthodes employées pour réaliser les pro- grammes d'amélioration des animaux domes- tiques.}

Dans différents pays, on a procédé à des distributions chez les éleveurs de reproducteurs nâles provenant d'établissements administratifs d'élevage; mais ces efforts, généralement trop dispersés et pratiqués, le plus souvent, sans méthode suivie, ont abouti à des résultats insignifiants.

La responsabilite de telles entreprises incombe, toutefois, aux gouvernements, qui se doivent d'en assurer le succès grâce à un contrôle sérieux des géniteurs et à une propagande efficace auprès des éleveurs.

Plars d'amélioration. - Chaque pays à son plan de travail plus ou moins précis, plus ou moins appliqué. Sont résumés ci-après ceux de l'Égypte, de l'Inde et du Kenya.

Égypte. - Le plan d'amélioration en cours vise l'élevage du buffle.

Dans un centre administratif, on réunit tout d'abord un troupeau important de sujets d'élite sélectionnés parmi le cheptel du pays en raison de leur vocation laitière et de leurs aptitudes reproductrices.

Les mâles issus de ce troupeau sont répartis sur le territoire, les uns dans des stations de monte officielles, les autres auprès des coopératives de fermiers, d'autres enfin chez certains éleveurs méritants. Dans ces deux derniers cas, ils restent cependant sous la surveillance des agents de l'administration.

Chaque reproducteur doit assurer des saillies gratuites dans un rayon de 10 kilomètres; il doit servir environ 150 femelles.

Il existe actuellement 73 stations de monte semblables et on envisage de porter ce chiffre à plus de 300. La mise en place de cette organisation nécessite la castration systématique et obligatoire des buffles non inscrits aux livres généalogiques. Cependant, cette mesure a été jugée inutile en raison de l'engouement manifesté par les éleveurs pour l'utilisation des étalons administratifs gratuits.

Dans chaque centre, un registre tenu par un agent de l'administration mentionne le nombre de femelles servies et ultérieurement le rendement laitier des génisses issues de l'étalon du centre.

Le Gouvernement égyptien envisage comme mesure d'avenir le recours de l'insémination artificielle, cette méthode de reproduction semblant convenir particulièrement à l'espèce bubaline.

A cet effet, l'équipement des villages en installations téléphoniques permettra des liaisons rapides 
entre les centres de production et les fermes d'utilisation.

Inde. - Le plan adopté par l'Inde présente quelques analogies avec le plan précédent. Toutefois, on estime ici que la castration obligatoire des mâles non inscrits est indispensable et que l'éparpillement des mâles provoque une discontinuité de l'action.

Le plan porte le nom de «Key Village Plan 》 et présente les caractéristiques essentielles suivantes :

$1^{\circ}$ On définit les races qui conviennent à une région déterminée.

$2^{\circ}$ La région est divisée en villages ou groupes de villages, possédant 500 femelles. Chaque secteur ainsi constitué reçoit le nombre de mâles nécessaires.

$3^{\circ}$ L'entretien de ces mâles reste sous le contrôle du Gouvernement.

$4^{\circ}$ La castration obligatoire des mâles non inscrits est décrétée dans tous les secteurs.

$5^{\circ}$ Chaque secteur doit servir de centre de démonstration en matière de conduite de troupeaux, de cultures fourragères, et doit porter son attention sur la commercialisation des produits de l'élevage en vue de procurer aux propriétaires des ressources susceptibles de les inciter à coopérer au plan.

Dans les régions de l'Inde favorables à l'élevage du mouton, un programme d'amélioration analogue a été engagé en vue de la production de la laine. Les béliers sont distribués gratuitement aux éleveurs, à charge pour chacun de ces derniers de céder un bélier de six mois choisi par l'État parmi les produits de la descendance (1).

Kenya. - Au Kenya, la répartition du cheptel en secteurs est basée non sur des villages ou groupes de villages sédentaires, mais sur des familles ou tribus vivant sur leurs terrains.

Ici encore, les taureaux issus des centres d'élevage sont distribués gratuitement aux éleveurs. Toutefois, dans les zones où sévit l'East Coast Fever. ces taureaux ne sont placés que dans des exploitations disposant d'installations de détiquage.

Certaines régions reconnues favorables ont pu permettre l'importation de races européennes. Mais le risque d'introduction des maladies provoquant la stérilité a conduit les pouvoirs publics à envisager l'insémination artificielle comme méthode de reproduction.

Dans les mêmes conditions, des races indiennes: Sahiwal et Red Sindhi ont été introduites, mais au sujet de ces introductions on redoute les conséquences pouvant résulter de l'apparition de nouveaux caractères défavorables tels que la diminution de la résistance aux maladies locales.

(1) A noter qu'une organisation analogue fonctionne depuis plusieurs années en Cochinchine pour l'élevage du porc.

\section{Questions diverses.}

$I^{\circ}$ La conférence a tenu à souligner d'une manière particulière les difficultés rencontrées, auprès" de nombreux gouvernements, par la mise en application des programmes d'amélioration des animaux domestiques.

Les résultats obtenus dans ce domaine'sont, en effet, beaucoup plus tardifs et moins rapidement spectaculaires que ceux que l'on peut obtenir en matière de production végétale.

Très souvent, des sommes importantes ont été gaspillées dans I'installation de fermes administratives modèles pour lesquelles n'avait pas été prévu un délai de rentabilité suffisamment prolongé et qui, faute de moyens financiers annuels suffisants, ont abouti à un échec.

Cette particularité devra être signalée à l'attention des fonctionnaires responsables de la répartition des crédits qui seront avertis du fait qu'un programme d'amélioration en matière d'élevage est plus lent et plus coûteux qu'un travail analogue en matière végétale.

$2^{\circ}$ Le délégué de l'Inde a fait ressortir les conditions spéciales que rencontre l'élevage dans ce pays, où le bcuf est considéré comme un animal sacré dont l'abatage est interdit par la religion locale. On a envisagé de créer de véritables camps de concontration pour grouper le bétail inutile et pour séparer les mâles des femelles en vue de tarir toute possibilite ulterieure de reproduction.

Le problème se présente d'une manière identique au Gouvernement du Pakistan.

En Birmanie, 'où la protection du bouf fait également partie des prescriptions éditées par les lois religieuses, on tolère toutefois l'abatage des animaux de plus de quatorze ans au profit de l'armée ou des hôpitaux.

30 Certains gouvernements, pour des motifs d'économie intérieure, ont jugé utile d'interdire l'exportation de leurs animaux vers d'autres pays.

L'allention de ces gouvernements doit être attirée vers les inconvénients que peut présenter une telle décision en matière de coopération zoo-économique internationale, en freinant le développement du programme d'amélioration animale dans les pays qui en sont victimes.

$4^{0}$ Le délégué du Pakistan souligne les erreurs commises dans l'établissement du recensement des animaux, dont les statistiques sont fréquemment très éloignées de la réalité. Il suggère que soit adoptée une méthode de recensement pratique qui permette d'exprimer des résultats aussi exacts que possiblc.

Il insiste également sur le fait que, dans certains pays, la nomenclature des races animales, exagérément compliquée par l'énumération de trop nombreuses variétés, gagnerait à être simplifiée.' 
$5^{\circ}$ Enfin, la conférence met l'accent sur la pénurie actuelle de techniciens, qui affecte de nombreux pays. Elle souhaite que les gouvernements intéressés prennent les mesures nécessaires pour le recrutement de spécialistes en matiere d'élevage et de physiologie animale.

\section{III. - RECOMMANDATIONS DE LA CONFÉRENCE}

La conférence, ayant considéré les différents problèmes fondamentaux dans le domaine de l'amélioration des animaux domestiques et ayant entendu les rapports des délégués des différents pays, recommande ce qui suit :

10 Que l'attention des gouvernements des territoires sous climat tropical soit attirée sur l'importance économique de l'élevage, tant dans le domaine de la fertilisation du sol que dans celui de l'alimentation humaine. Chaque gouvernement est invité à consacrer à son développement, qui constitue une cuvre de longue haleine, les moyens financiers ainsi que le personnel technique nécessaires.

$2^{\circ}$ En vue d'encourager les éleveurs à coopérer aux programmes d'amélioration du bétail, les gouvernements doivent assurer aux produits d'élevage des prix rémunérateurs.

30 Les gouvernements sont invités à former et recruter des techniciens qualifiés en nombre suffisant pour mener à bien les recherches de génétique, de physiologie et de pathologie animales, et a donner a ce personnel les moyens de travail et le standard de vie susceptibles de le retenir à l'œuvre.

La conférence émet le vœu que la F.A.O. veuille bien accorder ses bons offices pour la formation et l'entraînement de tels spécialistes.

Par ailleurs, les divers pays sont invités à une étroite collaboration dans ce domaine, soit en organisant l'échange de spécialistes d'un pays à l'autre soit en accordant des facilités réciproques aux chercheurs des pays étrangers.

$4^{\circ}$ Que les gouvernements prennent en considération l'importance des stations de recherches sur l'élevage travaillant en liaison avec les populations rurales.

$5^{\circ}$ La méthode d'amélioration basée sur la distribution de mâles sélectionnés est considérée comme très efficace. Toutefois, cette méthode doit avoir pour corollaire :

a) la castration obligatoire de tous les mâies non reconnus aptes à la reproduction, ou tout au moins leur éloignement des centres de reproduction;

b) aucun centre nouveau ne doit être créé si l'or. n'est assuré de pouvoir le réapprovisionner en reproducteurs mâles pendant au moins quatre générations. $3^{\circ}$ Le buffle joue, dans certains pays, un rôle important comme producteur de lait et comme animal de travail. Son amélioration zootechnique mérite de recevoir, de la part des gouvernements intéressés, la même attention que l'amélioration des autres especes domestiques.

$i^{\circ}$ L'insémination artificielle apparaît comme le moyen de pallier la disproportion qui existe entre les besoins de la reproduction et le nombre de mâles améliorateurs disponibles.

Cette méthode basée sur l'emploi de reproducteurs de valeur bien connue ne devra être utilisée que lorsque les conditions de milieu répondront aux exigences alimentaires des races amélioratrices utilisees.

La conférence constate que cette méthode ne constitue ni une fin en elle-même ni un remède a toutes les déficiences, mais invite, toutefois, les gouvernements à poursuivre dans ce domaine des recherches expérimentales et à étudier les possisilités d application de la méthode dans les conditions tropicales.

I.es possibilités de transporter le sperme à distance par voie aérienne doivent être exploitées au maximum. L'organisation devra permettre des foumitures régulières et continues de semences de bonre qualité.

Ia diffusion des resultats scientifiques sous forme de srochures de vulgarisation à des éleveurs. parfois illettrés, est inopérante; il convient ici de vulgariser l'information scientifique, soit par l'institution de fermes modeles de démonstration, soit par un service de propagande, comme aux U.S.A.

80 Four l'avancement des recherches de génétique et de niyysiologie, la création de laboratoires équipés de rratériel moderne et de personnel qualifié est nécessaire. Toutefois, la conférence souligne la récessité de ne pas se borner à installer quelques grands laboratoires dans les pays hautement industrialisés. Il faut s'efforcer de décentraliser les stations de recherches afin de les mettre à la portée des expérimentateurs opérant sur les lieux d'élevage.

$9^{\circ}$ Il est considéré comme nécessaire d'uniformiser dans tous les pays les unités de mesures pour la production de matières grasses, de viande, de travail, et pour l'utilisation de la ration alimentaire.

i. $0^{\circ}$ En matiere de prodiction laitière, les pays tropicaux devront chercher a definir les facteurs de correction inherents au milieu tropical, en vue de les comparer aux mêmes facteurs des régions tempérées.

i Io La détermination des caractères héréditaires ces animaux par l'examen des produits est facilitée, en Europe, grâce à une précocité supérieure. Audela de la cinquième génération, on considère que les caracteres héréditaires sont acquis.

Sous les tropiques, les animaux étant plus tardifs, 
cette détermination est plus longue à établir; en conséquence, la conférence émet le vou que les divers pays se consacrent à la recherche du délai minimum exigé pour la fixation des caractères héréditaires.

$12^{\circ}$ Il est recommandé que les pays accordent une attention particulière à la définition d'indices de sélection simples qui tiennent compte à la fois des caractères héréditaires et de leur importance économique.

$13^{\circ}$ Certains pays ayant éprouvé des difficultés à obtenir des renseignements exacts sur des reproducteurs importés, il est recommandé qu'en cette matière les divers pays appliquent une etroite coopération. Tout pays exportateur de races améliorées devra fournir aux pays importateurs tous les éléments d'information nécessaires.

1 Lo Certaines races indigènes, soumises à des croisements trop nombreux, sont en voie de disparition. La conférence émet le vœu que les gouvernements s'efforcent de préserver ces races, dans !eur état pur, en vue d'éventuelles expérimentations ultérieures.

$15^{\circ}$ Les gouvernements sont invités à définir les caractères essentiels de leurs races respectives, et à les fournir au catalogue mondial de bétail en voie de réalisation par la F.A.O. én vue d'une utile coopération internationale.

$16^{\circ}$ Considérant que le travail d'amélioration effectué sur les races des pays tropicaux par le procédé de l'importation des géniteurs a généralement été conduit dans un but commercial, la conférence recommande que les divers gouver- nements s'astreignent à poursuivre ces essais sur des bases plus scientifiques permettant d'établir des normes faciles à interpréter rapidement.

$17^{\circ}$ Certaines races indigènes s'étant révélées réfractaires à certaines affections enzootiques, il est recommandé que les territoires échangent sur ce point toutes les informations utiles.

$18^{\circ}$ La multiplicité des races indigènes, souvent basées sur des caractères différentiels insignifiants, amène la conférence à souhaiter que chaque pays établisse une monographie rigoureuse de ses races et supprime dans sa nomenclature les variétés dépourvues d'intérêt.

190 La conférence souligne la nécessité d'un recensement annuel rigoureux du cheptel de chaque pays, trop souvent négligé jusqu'ici. Il serait souhaitable que ces statistiques soient fournies en prévision du recensement mondial établi par la F.A.O. pour 1950.

$20^{\circ}$ Devant le danger que présente la surcharge des pâtures pour la conservation des sols, chaque gouvernement aurait intérêt à évaluer l'effectif maximum de son cheptel compatible avec la superficie de ses terres disponibles, et à se débarrasser des animaux en excédent.

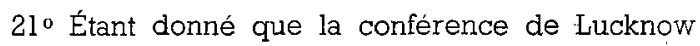
portant essentiellement sur la génétique et la physiologie n'a pas traité les questions d'alimentation et d'abreuvement, éléments dominants en matière d'élevage, il est recommandé qu'une autre conférence traitant de l'alimentation et de l'abreuvement du bétail en milieu tropical soit ultérieurement réunie. 